\title{
Islands Pflanzenwelt zwischen Feuer und Eis, Teil 2: Vegetationstypen
}

\author{
Karin Steinecke \& Hilke Steinecke
}

\begin{abstract}
Iceland features diverse landscapes with mountains, extended coastlines, glaciers, lava planes and (active) volcanoes. Plants are specifically adapted to the diverse types of habitats and unique vegetation types characterize individual landscapes. Iceland's natural landscape appears uniquely untouched and pristine, yet environmental problems do exist. Soil erosion resulting from overgrazing by vast flocks of sheep is an issue of major concern. The main vegetation types of Iceland and some of its most characteristic plants are summarized.
\end{abstract}

\section{Zusammenfassung}

Island hat eine abwechslungsreiche Landschaft mit Bergen, langen Küsten, Gletschern, Lavafeldern und (aktiven) Vulkanen. An die unterschiedlichen Standorte sind verschiedene Pflanzen angepasst und es haben sich diverse Vegetationstypen ausgebildet. Trotz der gigantischen, noch sauber und intakt erscheinenden Natur gibt es in Island eine Reihe von Umweltproblemen. Erosion aufgrund von Überbeweidung durch zu viele Schafe ist ein Hauptproblem. Die verschiedenen Vegetationstypen mit ihren charakteristischen Pflanzen sowie ihre Gefährdung werden zusammengefasst.

\section{Vielseitige Landschaft}

Island hat eine etwa $5000 \mathrm{~km}$ lange Küstenlinie, die besonders im Westen, Norden und Osten durch Fjorde, Steilküsten, Schären oder Buchten geprägt ist. An der flachen Südküste Islands tragen die großen Gletscherflüsse ge- waltige Sedimentmengen ins Meer. Hier finden sich ausgedehnte schwarz-sandige Strände, Sanderflächen und Kiesbänke, die eine dynamische Ausgleichsküste bilden. Die Anlage von Häfen ist an dieser dynamischen Küste nicht möglich, ebenso dauerte es bis in die

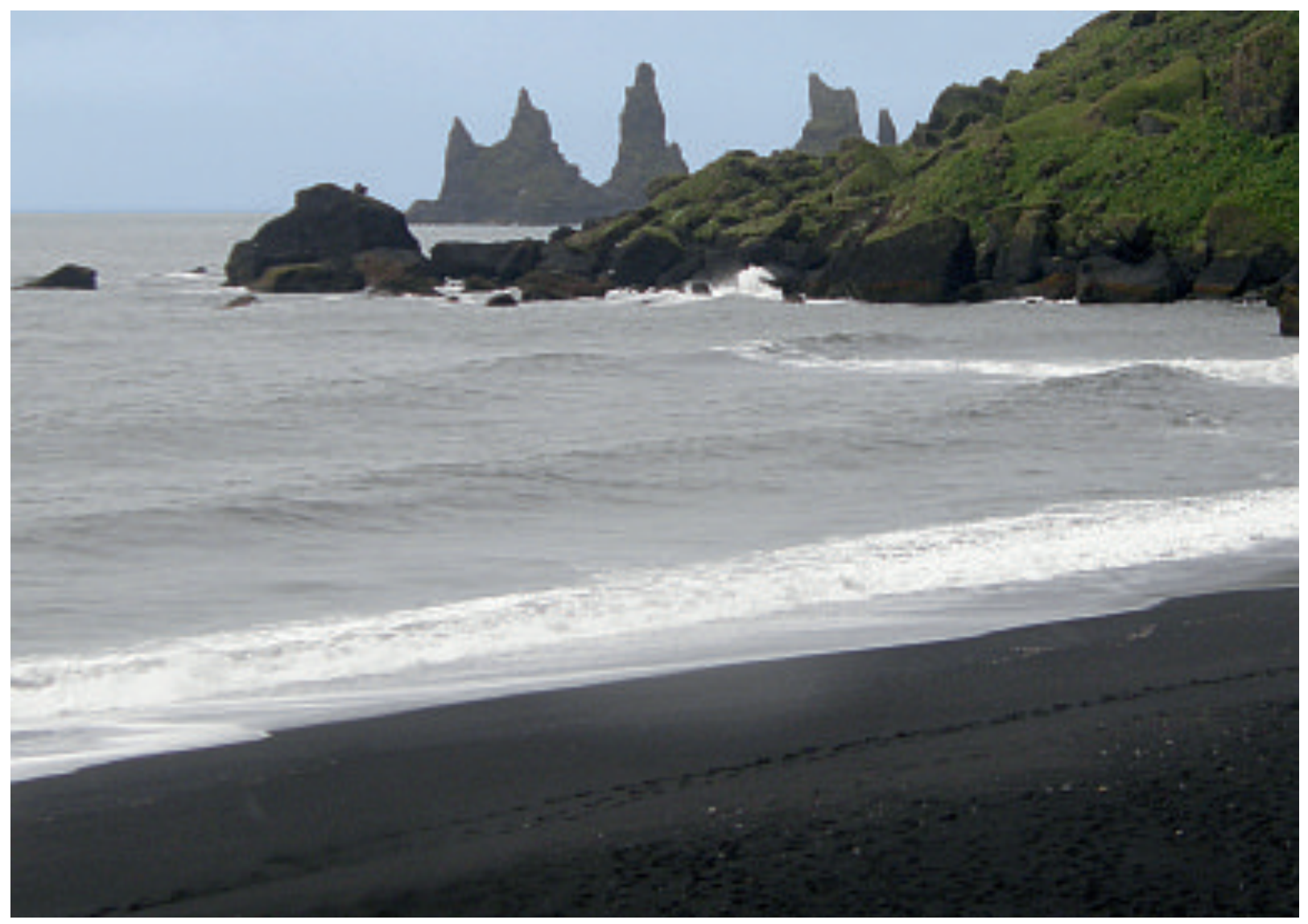




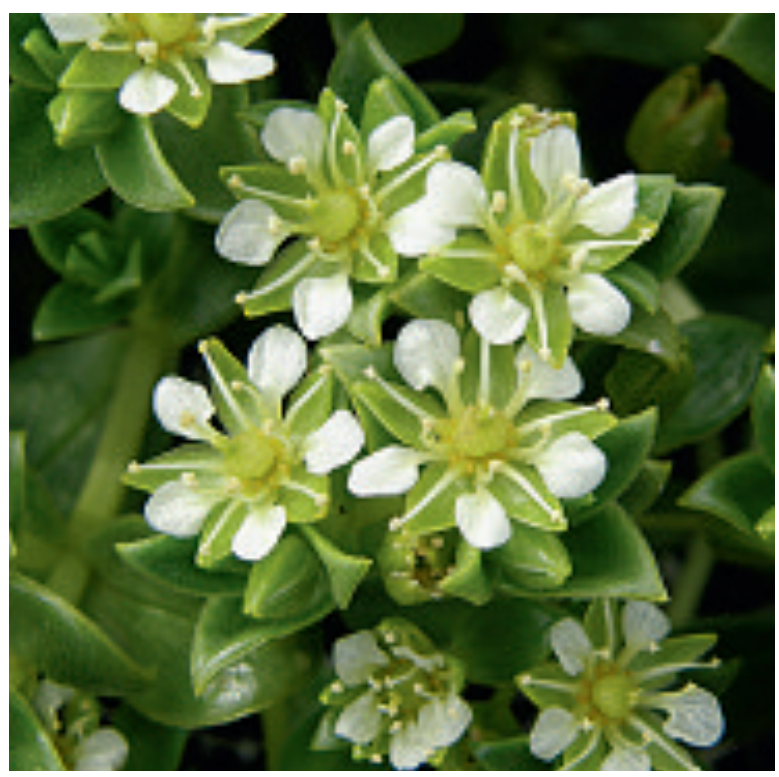

1960er Jahre, bis im Bereich der großen Sanderflächen Islands die die Insel umrundende Ringstraße geschlossen werden konnte. Bei Vulkanausbrüchen mit nachfolgenden Gletscherläufen in Südisland kommt es immer wieder einmal zu Beschädigungen der großen Brückenbauwerke.

\section{Spülsäume und Dünen}

An den flachen Strandabschnitten z.B. am Fuße von Kap Dyrhólaey, der Südspitze Islands, können sich oberhalb der Hochwasserlinie Spülsaum- und Dünengesellschaften entwickeln, die denen unserer Nordseeküste nicht unähnlich sind (Thannheiser 1985, 1987, 1988). Im Spülsaumbereich gedeihen salzverträgliche annuelle Arten wie die Salzmiere (Honckenya peploides) und der Arktische Meersenf (Cakile arctica). Letzterer ist essbar und hat kohlartigen Geschmack. Die Dünenbildung wird in erster Linie durch den Strandroggen (Leymus arenarius) initiiert, der aber auch an sandig-schottrigen Standorten im Hochland

Abb. 1 (Seite 102): An der Südküste Islands gibt es lange, schwarze Lavastrände.

Abb. 2 (links): Honckenya peploides.

Abb. 3 (rechts): Reste natürlicher Birkenwaldvegetation bei Hraunfossar, West-Island.

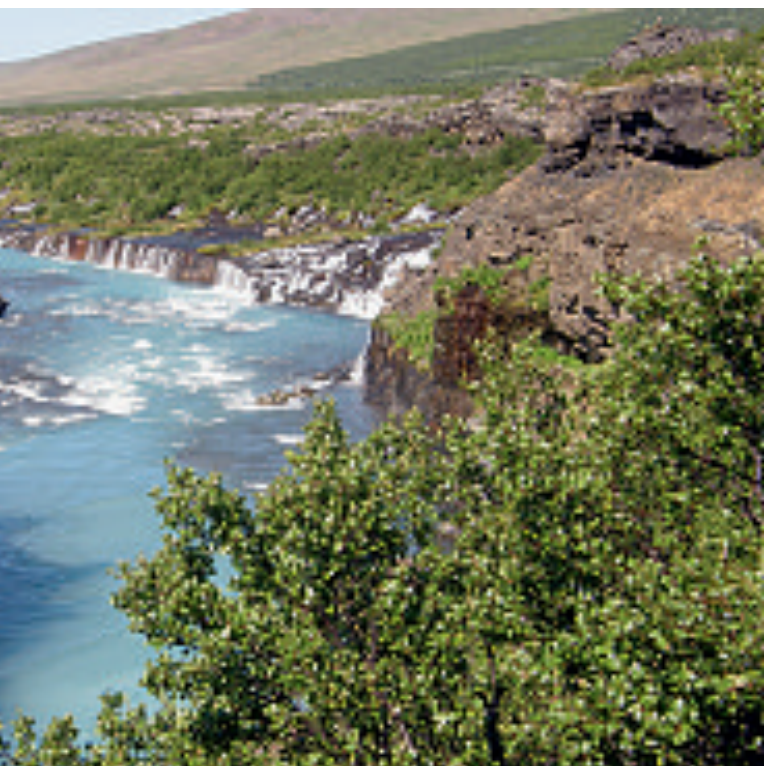

vorkommt. Auf Dünen- und Sandflächen macht sich auch die Strand-Platterbse (Lathyrus japonicus) breit. Sie kommt, entgegen ihres wissenschaftlichen Namens, an den Küsten des Nord-Atlantiks vor und wird häufig von Schafen gefressen. Das Strand-Blauglöckchen (Mertensia maritima) ist durch seine sukkulenten, bläulich überlaufenen Blätter und die blauen glockenförmigen Blüten gekennzeichnet. Die kriechende bis aufrecht wachsende Staude ist an Küsten des Nord-Atlantiks verbreitet. Aufgrund ihres austernähnlichen Geschmackes wird sie auch Austernpflanze genannt und regional als Würzpflanze genutzt. An Fels- und Steilküsten sind orangefarbene Flechten der Gattung Caloplaca sowie die Strandnelke (Armeria maritima) typisch.

\section{Birkenwälder}

Auf Island kommen natürliche Birkenwälder mit Betula pubescens, Sorbus aucuparia und Weidenarten in der Baum- und Strauchschicht vor. Mit maximalen Baumhöhen von 3 Metern und einer teilweise geringen Baumdichte erscheint dieser Vegetationstyp kaum den Ansprüchen eines echten Waldes zu entsprechen. Dennoch gilt der isländische Birkenwald als einer der artenreichsten Biotope dieser Insel. Mittlerweile auf kleine Areale zurückgedrängt, sind die Reste des natürlichen Waldes streng geschützt. 

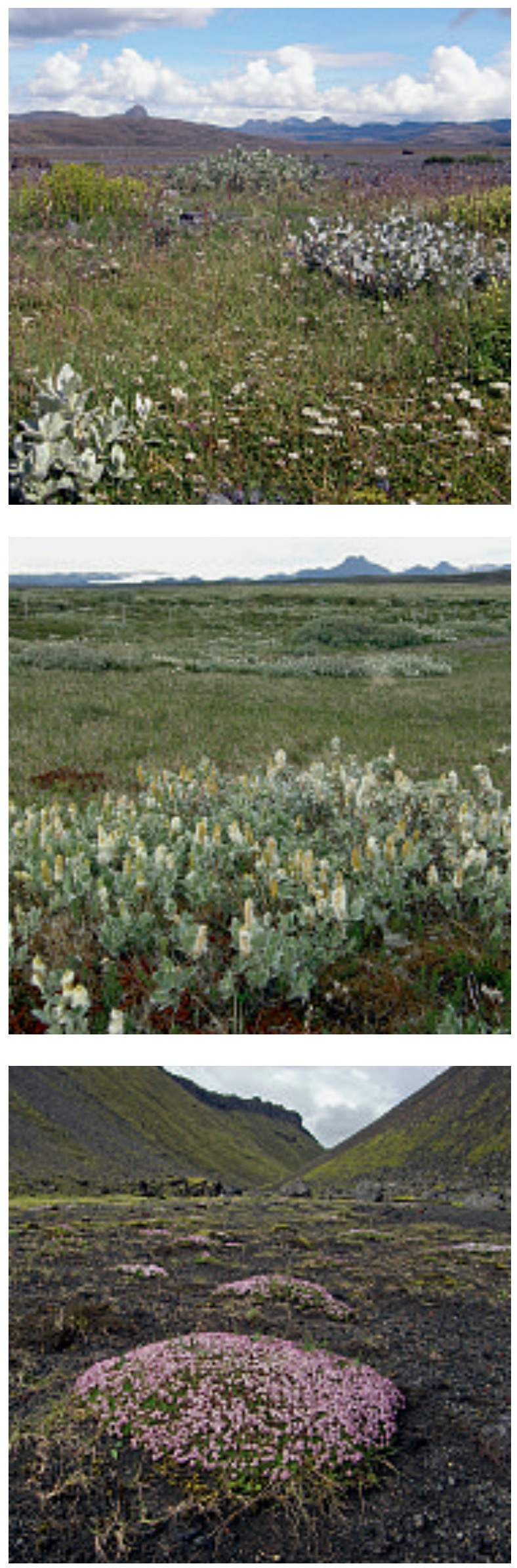

\section{Zwergstrauchgesellschaften}

Von der Küste bis in eine Höhe von etwa 400 m treten Zwergstrauchheiden mit verschiedenen Heidekrautgewächsen, darunter auch Heidelbeere (Vaccinium myrtillus), Rauschbeere ( $V$. uliginosus) und Krähenbeere (Empetrum nigrum), auf. Diese Heiden gehen in größerer Höhe in moos- und flechtenreiche GebirgsZwergstrauchtundren über. Unter den Gehölzen der Zwergstrauchformationen sind vor allem die nur bis $60 \mathrm{~cm}$ hohe Zwerg-Birke (Betula nana) und vier verschiedene Weiden-Arten zu nennen. Die kleinste von ihnen ist die auch in den Alpen weit verbreitete Kraut-Weide (Salix herbacea), deren nur einige Zentimeter hohe Triebe meist nur wenige Blätter und die Blütenstände ausbilden. Wollige Weide ( $S$. lanata) und Arktische Grauweide (S. callicarpea) sind durch sehr wollige Samen gekennzeichnet; da sie hybridisieren, sind diese beiden Arten nur schwer auseinanderzuhalten. Die Grüne Weide (Salix phyllicifolia) ist neben der Birke der wichtigste Waldstrauch. Die Zwergstrauchheiden Islands sind zum einen natürlichen Ursprungs, stellen in weiten Teilen aber auch Degenerationsstadien des subpolaren Birkenwaldes nach intensivem Verbiss und Tritt durch die Schafe dar (Glavion 1986, Hövelmann 1995).

Bei noch weitergehender Beweidung geht die Zwergstrauchheide schließlich in die offene sog. Melur-Vegetation über, die sich natürlicherweise auch im zentralen Hochland auf trockenen Lockersubstraten findet. In den wüstenhaften Gebieten des Hochlandes herrschen Bodentrockenheit und starke Winde, die zu Umlagerungen des lockeren Bodens führen. Unter diesen schwierigen Bedingungen können sich in den Dauerpioniergesellschaften des isländischen Hochlandes also vor allem gedrungene Polsterpflanzen halten, wie sie auch aus den Alpen bekannt sind. Im Winter sind die niedrigen, kompakten Pflanzen durch den Schnee geschützt;

Abb. 4 (oben): Zwergstrauchvegetation im Hochland.

Abb. 5 (Mitte): Salix lanata.

Abb. 6 (unten): Polsterflur mit Silene acaulis in Landmannalaugar, Süd-Island. 
im Sommer können die festen Polster dem Wind und der Kälte Widerstand leisten, zudem sammeln sich in den Polstern durch verrottende Blätter Nährstoffe an. Häufig haben die Polsterpflanzen lange Pfahlwurzeln. Besonders auffällig ist das Stängellose Leimkraut (Silene acaulis); die Polster sind so dicht von den kleinen rosa Blüten überzogen, dass die Blätter kaum noch zu erkennen sind. In Notzeiten wurden die Wurzeln ausgegraben und gegessen. Die verwandte Art Silene uniflora blüht hingegen weiß und ist wie auch die bereits erwähnte Grasnelke (Armeria maritima) ebenfalls im Hochland anzutreffen.

\section{Moore}

In den Niederungen Islands bilden sich häufig durch Verlandung von Seen und Tümpeln Niedermoore aus. Dort findet man vor allem Seggen, Wollgras (Eriophorum angustifolium und E. scheuchzeri), Binsen und das SumpfHerzblatt (Parnassia palustris). Von den karnivoren Arten ist hier das Fettkraut (Pinguicula vulgaris) vertreten. Häufig ist zudem die AlpenTrauerblume (Bartsia alpina) mit ihren schwarzvioletten Blüten. Es handelt sich wie beim Klappertopf und dem Augentrost um einen Halbparasiten. Das kühle Klima und die fehlende Zersetzung durch Mikroorganismen führen zur Bildung von mächtigen Torflagen. Torf war früher neben Schafsdung der einzige Brennstoff und auch wichtiges Baumaterial für die Häuser und Kirchen der Isländer. Torfhäuser sind heute aber fast nur noch in Freilichtmuseen zu sehen. Durch häufigen Frostwechsel entstehen Moorböden dicht an dicht liegende kleine Frosthügel von mehreren Dezimetern Ausdehnung, die selbst dann erhalten bleiben, wenn die Moore durch Drainage in Wiesenland überführt werden. Da diese sog. Buckelwiesen wegen der Gefahr des Stürzens von Pferden und Kühen nicht als Viehweide

Abb. 7 (oben): Armeria maritima.

Abb. 8 (Mitte): Cardamine nymanii.

Abb. 9 (unten): Eriophorum scheuchzeri.
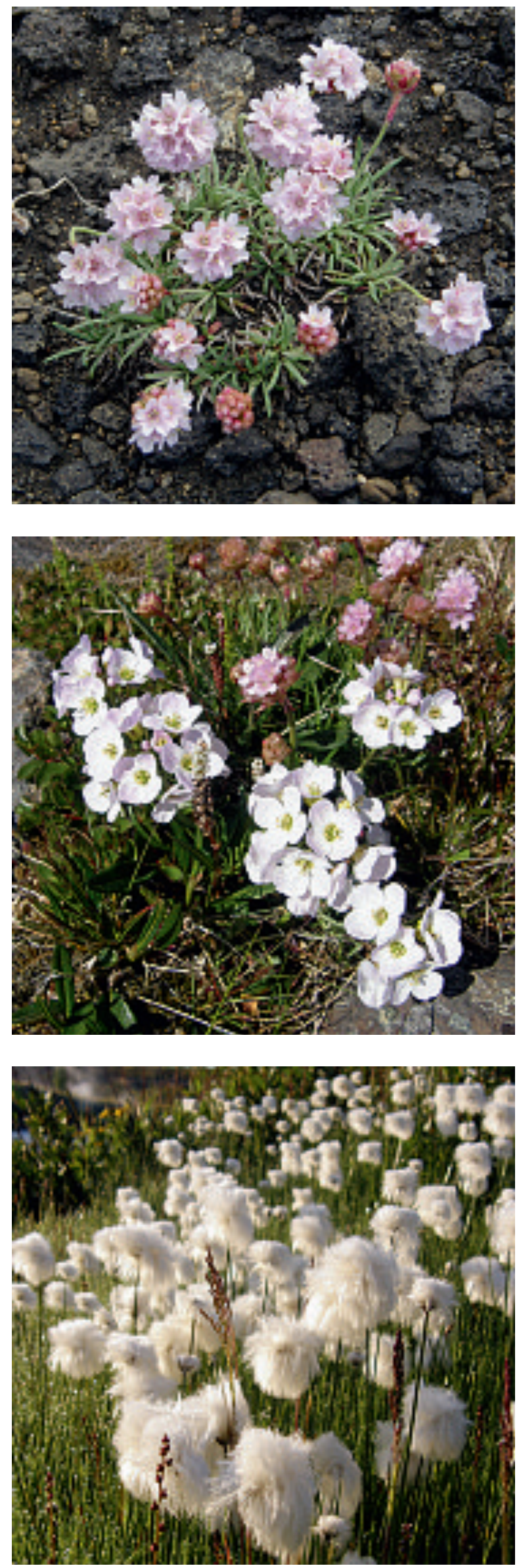

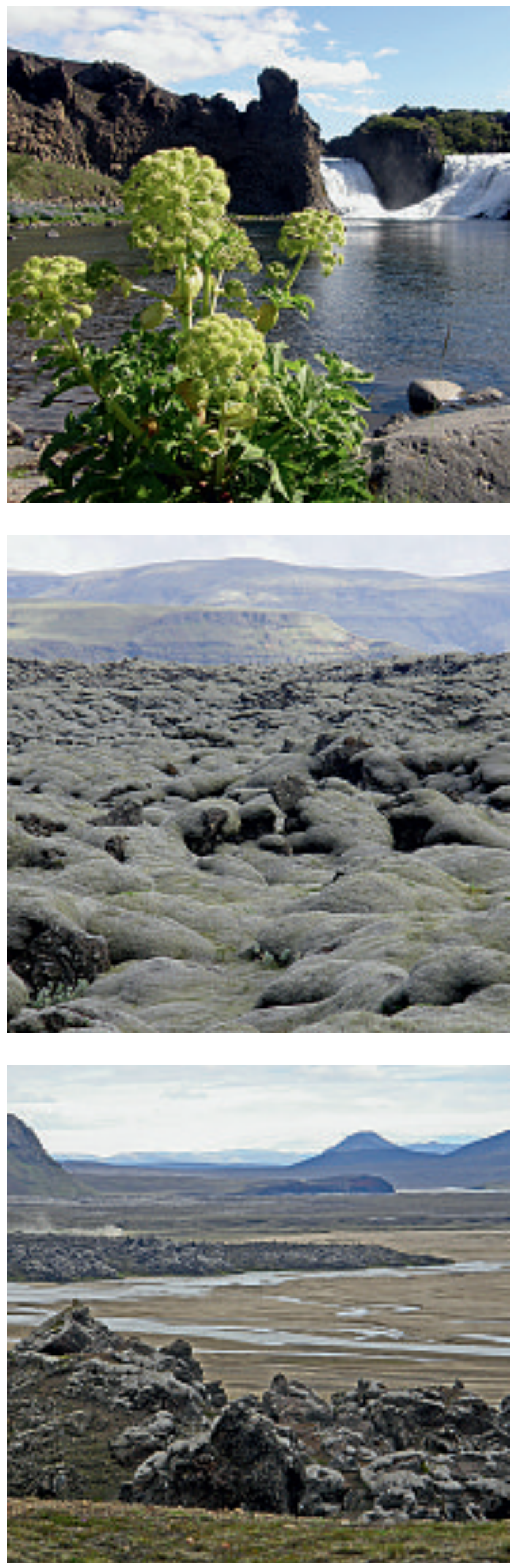

geeignet sind, werden sie häufig umgebrochen. Aber selbst dann können die Buckel nach einigen Jahren wieder neu entstehen. Die bis in die 1980er Jahre ständig zunehmende Drainage von Moorflächen zur Gewinnung von landwirtschaftlicher Nutzfläche konnte erst in den letzten Jahren auf Island allmählich eingedämmt werden. Heute stehen viele Moorflächen bereits unter Schutz.

\section{Pflanzenwelt der Wiesen, Ufer und Schluchten}

An feuchten Stellen und Bächen gedeihen oft große Bestände der Sumpfdotterblume (Caltha palustris). An Seeufern, Bachläufen oder in Schluchten ist die große Engelwurz (Angelica archangelica) eine häufige und auffällige Pflanze. Charakteristisch sind ihre halbkugeligen, grünen doldigen Blütenstände sowie die aufgeblähten Blattscheiden. Allgegenwärtig ist auch die Bach-Nelkenwurz (Geum rivale) mit ihren nickenden, rötlichen Blüten. Bei uns ist sie aufgrund von Entwässerungsmaßnahmen stellenweise sehr selten geworden und wurde deshalb zur Pflanze des Jahres 2007 gekürt. Auf feuchten Wiesen findet man zudem häufig Cardamine nymanii, das unserem heimischen WiesenSchaumkraut (Cardamine pratensis) sehr ähnlich sieht. An Felsen, auf kiesigen Böden, auch auf Bergkuppen und im zentralen Hochland wachsen Polster des Gegenblättrigen Steinbrechs (Saxifraga oppositifolia). Die immergrünen Blätter ertragen Temperaturen bis $-40^{\circ} \mathrm{C}$ ohne Schaden. Die für die kleinen Pflanzen überdimensional großen, purpur-roten Blüten haben eine große Signalwirkung und locken die Bestäuber besonders effektiv an. In ganz Island verbreitet ist die Silberwurz

Abb. 10 (oben): Angelica archangelica am Ufer des Wasserfalls Hjálparfoss in Süd-Island.

Abb. 11 (Mitte): Mit Zackenmützenmoos dicht bewachsenes Lavafeld in Süd-Island.

Abb. 12 (unten): Gletscherflüsse mit wechselndem Lauf prägen im Hochland vielerorts die Landschaft.

Abb. 13 (Seite 107): Altes Torfsodenhaus im Freilichtmuseum von Glaumbær, Nord-Island. 


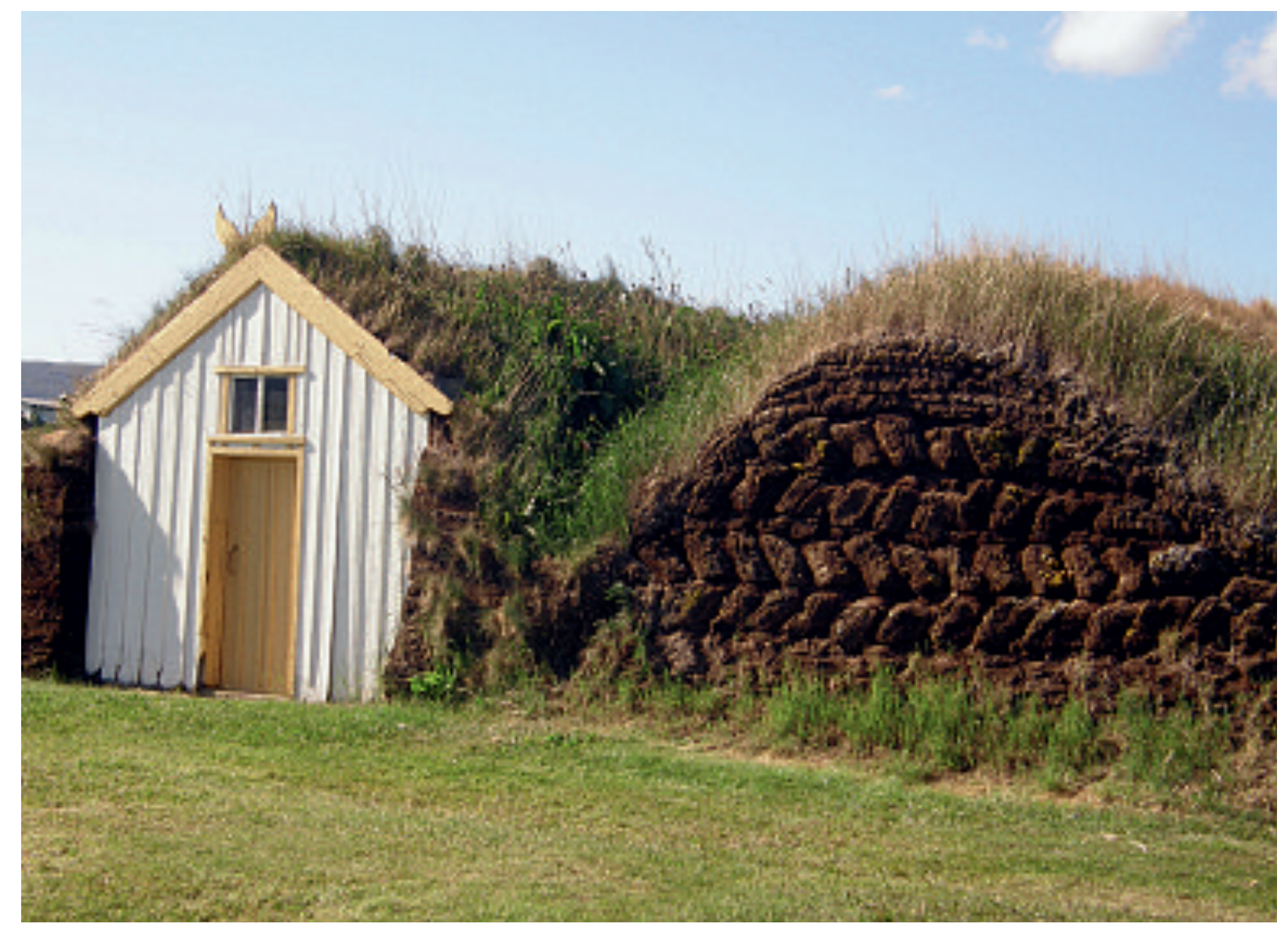

(Dryas octopetala). Die Ausbreitung ihrer Samen ist an den Wind angepasst. Wie bei Küchenschelle oder Waldrebe bleiben die langen behaarten Griffel an der Frucht erhalten und dienen als Flugorgan.

\section{Leben an Extremstandorten}

An Extremstandorten wie Lavaflächen und Gletschervorfeldern können sich nur Spezialisten halten. Moose und Flechten sind die ersten Besiedler und Wegbereiter für höhere Pflanzen. Sie sind austrocknungsresistent und können auf dem kargen Boden überleben. Sie sammeln Nährstoffe an, so dass später Substrat für anspruchsvollere Gewächse vorhanden ist. Dieser Pioniervegetation folgen die höheren Pflanzen. Besonders in Küstennähe können auch Vögel den Weg für die Ansiedlung von Pflanzen bereiten. Wenn Vögel immer wieder die gleichen Steine als Aussichtswarte nutzen, hinterlassen sie auf dem zunächst nackten Stein nicht unerhebliche Mengen an nährstoffreichem und Wasser speicherndem Guano. Darauf siedeln sich dann Gräser und andere Pflanzen an. Dieses Phänomen ist sehr schön in Lavafeldern oder an den Pseudokratern bei Kirkubæerklaustur an der Südküste nachzuempfinden. Auf den mehrere Meter hohen kegelförmigen Hügeln befinden sich auf ihren Spitzen etwa $50 \mathrm{~cm}$ hohe kissenartige, grasbewachsene Belege. Oft können darauf dann tatsächlich Vögel (z.B. Brachvögel, Möwen, Austernfischer) beobachtet werden.

Wenn ein Ascheregen auf ein von einer Pflanzendecke bedecktes Gebiet niedergeht, ist es erstaunlich, wie robust manche Gewächse sind und durch die Asche dem Licht entgegenwachsen. Sehr schön war dies in jüngster Zeit in Pórsmörk zu beobachten. Hier ging im Frühjahr 2010 reichlich Eyjafjalla-Asche nieder und bedeckte die Vegetation schneeartig mit einer dicken grauen Schicht. Aber schon wenige Wochen nach dem Vulkanausbruch trieben die ersten Pflanzen wieder aus, erste Blättchen und sogar vereinzelte Blüten waren zu sehen. Zu den ersten Arten, die „wie Phoenix aus der Asche“ 


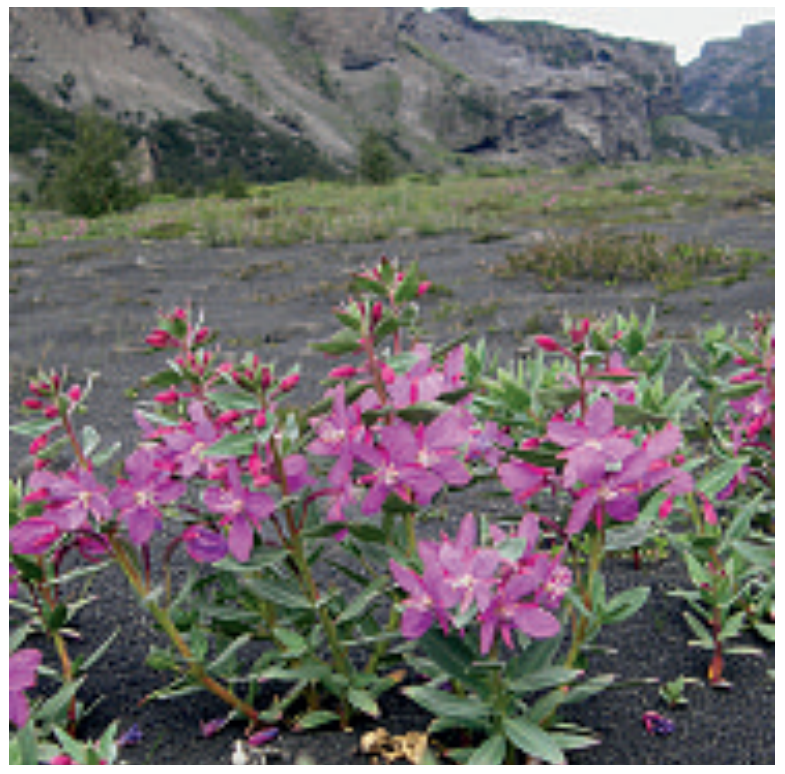

erschienen, gehörten Kleiner Frauenmantel (Alchemilla alpina), Stängelloses Leimkraut (Silene acaulis), Echtes Labkraut (Galium verum), Woll-Weide (Salix lanata) und Rauschbeere (Vaccinium uliginosum).

In Gletschervorfeldern und von Gletschern gesäumten Tälern verändern die großen Gletscherflüsse häufig ihr Bett, so dass sich auf den Sanderflächen zwischen dem Geröll nur einige wenige Pflanzen permanent halten können. In steinigen Flussbetten und auf Geröllhalden bildet das Arktische Weidenröschen (Epilobium latifolium) oft große Bestände. Seine bis $4 \mathrm{~cm}$ breiten pinkfarbenen Blüten sind sehr auffällig. Als eine der schönsten Wildpflanzen Islands zierte es über Jahre die Verpackungen von Milch. Auch in den Alpen besiedeln Weidenröschen ähnliche ökologische Nischen. Dort ist es Fleischer's Weidenröschen (Epilobium fleischeri), ebenfalls eine Art mit relativ großen purpurroten Blüten. In den Alpen sowie in Island findet sich auf Kieshalden und im Geröll der Gletscherflüsse auch reichlich der Fetthennen-Steinbrech (Saxifraga aizoides).

Ein Kontrast zu diesen kalten Standorten stellen heiße Quellen und blubbernde Schlammlöcher dar. Auch in heißen Quellen ist noch Leben möglich. Abhängig von der Temperatur siedeln sich in ihnen Bakterien und Algen an. Ihre bunten Farben, die von Hellgelb über Orange bis zu Dunkelgrün reichen, ver- danken die heißen Quellen der Kombination aus Mineralien und Bakterien (SchutzBach 1999). Besonders auffällig ist die milchig blaue Farbe mancher heißer Quellen und Seen. Auch die berühmte blaue Lagune auf Reykjanes eigentlich ein im Lavafeld gelegener und mit dem warmen „Abwasser“ eines Geothermalkraftwerks gespeister Tümpel, der als Schwimmbad ausgebaut wurde-, zeigt diese Farbe. Das $37-29^{\circ} \mathrm{C}$ warme Wasser der blauen Lagune ist eine Mischung aus Salz- und Süßwasser, enthält hohe Anteile an KieselerdeSchlamm, Mineralsalzen und blaugrünen Algen. Das Blau wird durch die Kombination von Algen und Kieselsäure verursacht. Vor allem durch die Kieselsäure werden die blauen Lichtanteile besonders intensiv reflektiert. Dadurch erscheint der für uns ungewöhnliche Farbeindruck, ähnlich wie beim Gletschereis. Kieselerde und Mineralsalze dieser Lagune sollen heilend bei Gürtelrose und Erkrankungen der Haut wirken und werden als Cremes und Lotion in viele Länder der Welt exportiert.

\section{Gefährdung von Flora und Vegetation}

Island gilt weithin als ein Land unberührter Natur und ohne Umweltprobleme. Dies ist aber sicher eine falsche Vorstellung für ein hoch entwickeltes westliches Industrieland, das beispielsweise mit dem weltweit zweithöchsten Pro-Kopf-Stromverbrauch und der Weltrangposition 10 der Zahl der PKW pro 1000 Einwohner aufwarten kann. Noch vor wenigen Jahren gab es in Island kaum Kläranlagen oder entsprechend gegen Sickerwasser abgedichtete Deponien. Auch das bleifreie Benzin wurde von allen europäischen Ländern in Island als letztes eingeführt (Steinecke 1993, 1995; Gustafsson \& Steinecke 1995). Die sich wegen der niedrigen Energiepreise ansiedelnde Schwerindustrie (Aluminiumverhüttung) belastet die Umwelt durch Emissionen. Die Anlage und der Ausbau

Abb. 14 (oben): Epilobium latifolium.

Abb. 15 (Seite 109): Geothermalfeld Námaskarð (Nord-Island) mit dampfender Fumarole und milchig blauer heißer Quelle. 
von Wasserkraftwerken und Geothermalkraftwerken zerstören zudem einzigartige Lebensräume und Naturdenkmäler.

Als größtes Umweltproblem wird aber nach wie vor die fortschreitende Bodenerosion in Island angesehen (GLAwion 1987), die mit der Besiedlung durch die Wikinger begann und durch die Tritt- und Verbissschäden am natürlichen Birkenwald durch nicht native Großherbivoren ausgelöst wurde. Heute tragen zur Erosion neben der Beweidung z. B. auch Offroad-Fahrten mit schweren Geländewagen durch Einheimische und durch Touristen bei. Nur 11,3\% der Landesfläche zeigen aktuell keine Erosionsschäden, geringe Schäden sind auf 26,0 \% der Fläche festzustellen, deutliche Schäden auf 22,5\% und starke Beeinträchtigungen auf $11,0 \%$. Etwa 6,2\% aller Böden sind sogar sehr stark durch Erosion gefährdet (ScHMID 1991). In einigen Bereichen werden sogar Erosionsraten von bis zu $100 \mathrm{~m} / \mathrm{Jahr}$ in der Vertikalen bzw. $10 \mathrm{~cm} / \mathrm{Jahr}$ in der Horizontalen er- reicht. Zur Einschränkung der Bodenerosion werden mobile Substrate an gefährdeten Stellen durch technische Hilfsmittel (Netze, Reisigzäune etc.) gebunden sowie flugzeuggestützte Aussaaten von Gräsern (u. a. Leymus arenarius) durchgeführt. Durch Düngung und andere Bodenverbesserungsmaßnahmen wird versucht, die Entwicklung einer geschlossenen Pflanzendecke zu fördern.

\section{Aufforstung}

Vielerorts wird auch aufgeforstet, allerdings sind die aufgeforsteten Bäume zu 70\% nicht heimisch. Häufige Forstbäume sind auf Island u. a. Picea glauca, P. engelmannii, P. sitchensis, P. abies, Larix sibirica, Pinus contorta, Populus trichocarpa, Betula pendula und Ulmus glabra. Gegenüber der heimischen Birke haben diese Arten den Vorteil einer hohen Wuchsleistung, der Nutzholzgewinnung sowie der Weihnachtsbaumproduktion; die Florenverfälschung auf Island wird dadurch jedoch weiter verstärkt.

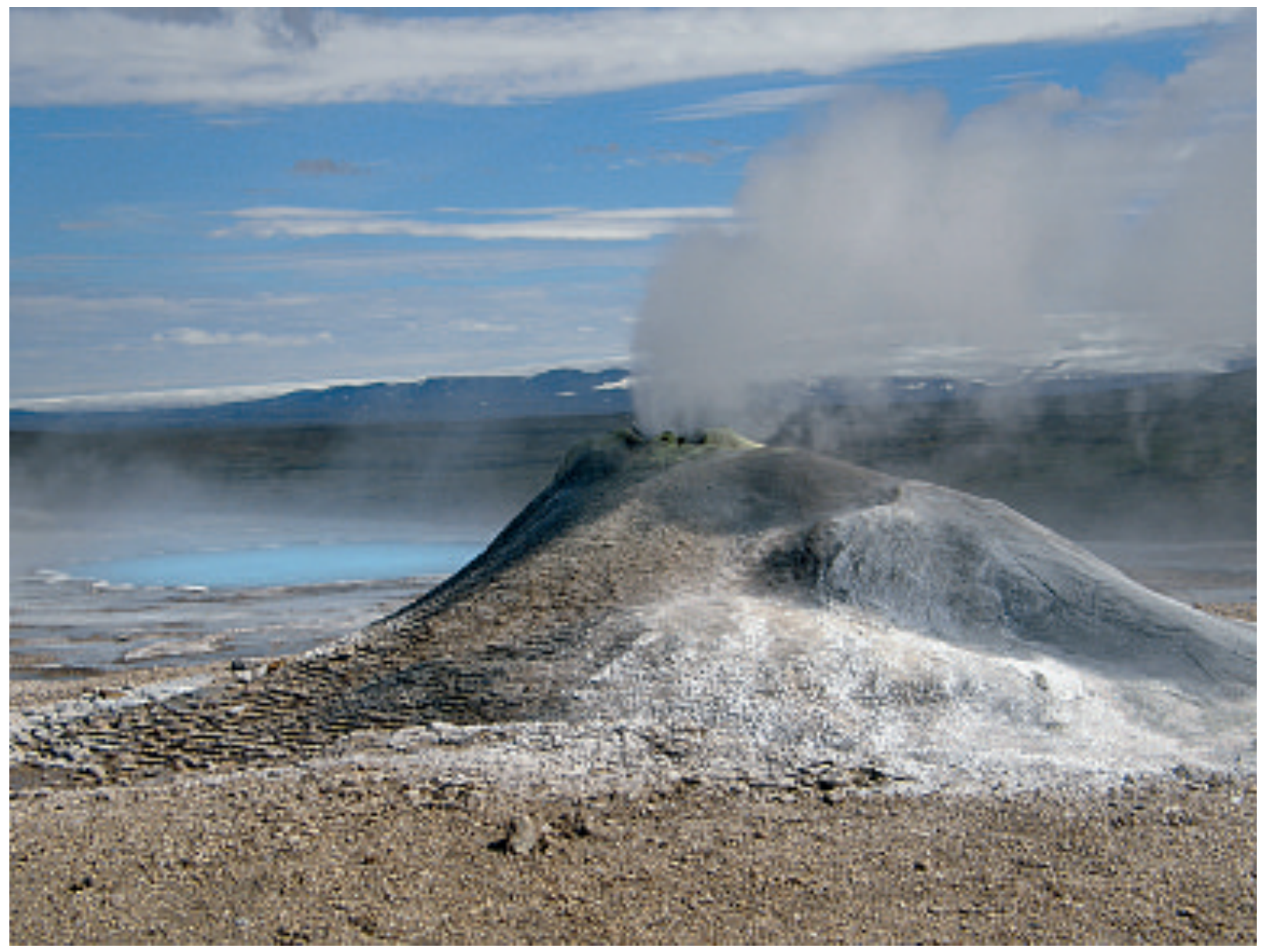




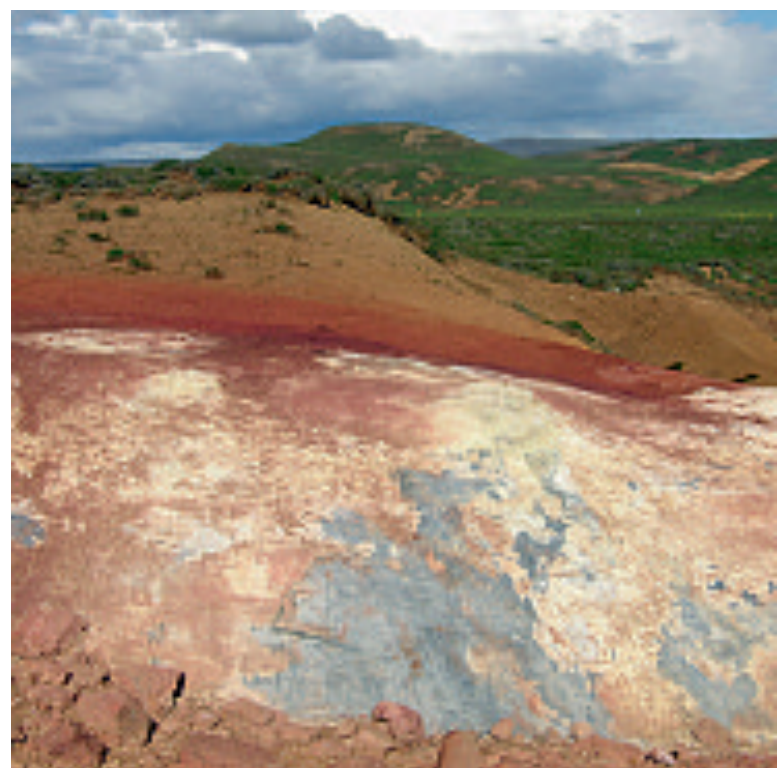

Erste Wiederaufforstungsprojekte gibt es in Island bereits seit 1899, gezielter seit den 1930er Jahren. In diesen mittlerweile fast 80 Jahre alten Wäldern (z. B. in Hallormstaður in Ost-Island) finden sich mittlerweile Bäume von über 15 m Höhe. Sie sind der ganze Stolz der Isländer. Im Mittel werden seit 1990 jährlich Anpflanzungen von 3 bis 4 Mio. Setzlingen auf etwa 1100 ha durchgeführt. Bis zum Jahre 2040 sollen etwa 2,4\% der Landesfläche wieder mit Wald bestanden sein.

So erfolgreich mittlerweile auch die Wiederaufforstungsprojekte sind, muss dennoch auch langfristig gegen eine fortschreitende Erosion gearbeitet werden. Hierzu müssen grundlegende Umdenkensweisen in der Landwirtschaft erfolgen. Die Zahl der Schafe müsste dazu weiter reduziert werden und die Haltung von einer freien Fernweide auf eine Umtriebsweide in eingefriedeten Arealen umgestellt werden. Bis dahin wird aber sicher noch einige Zeit vergehen. Aufgrund der auch in Island zu erwartenden Klimaerwärmung wird es in den nächsten Jahren sicher zu einer weiteren Einwanderung von

Abb. 16 (links): Farbenspiel an heißen Quellen, Námaskarð.

Abb. 17 (rechts): Säcke mit Saatgut, das auf erosionsgefährdete Flächen ausgebracht wird, um die Erosion zu dämmen.

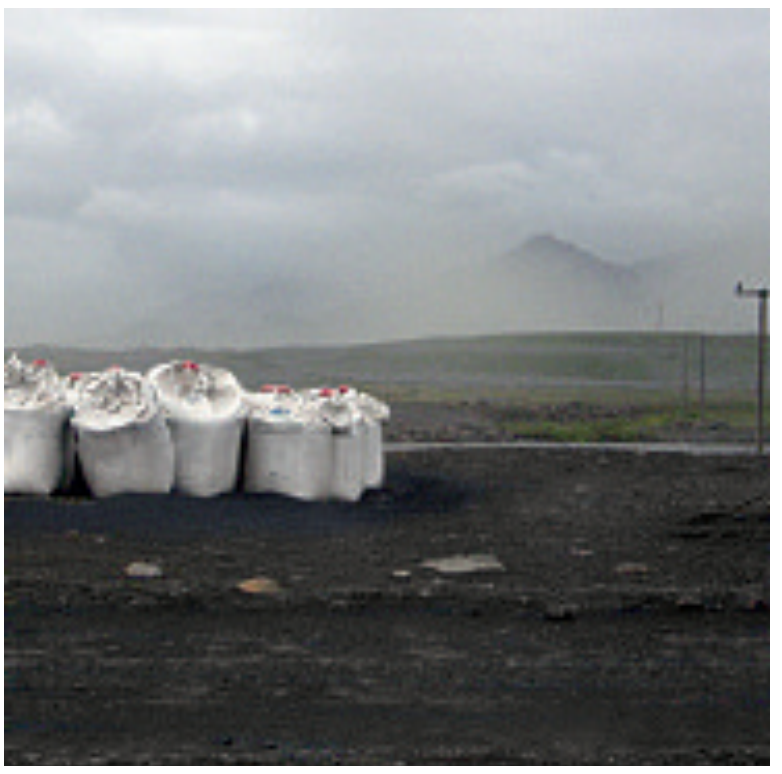

exotischen Pflanzen nach Island kommen. Die Isländer freuen sich jedenfalls darauf, vielleicht in Zukunft wieder die Gerste für ihr eigenes Bier selber anbauen zu können.

\section{Literatur}

GLAWION, R. 1986: Progressive und regressive Sukzessionen des isländischen Birkenwaldes. Berichte aus der Forschungsstelle Neðri Ás Hveragerði Nr. 47.- Hveragerði. Glawion, R. 1987: Leistungspotential und anthropogene Gefährdung subpolarer Ökosysteme am Beispiel Islands. - Verhandlungen des Deutschen Geographentages 45: 361-369.

Gustafsson, L. E. \& Steinecke, K. 1995: Airborne contaminants and their impact on the city of Reykjavík, Iceland. - Sci. Total. Environ. 160/161: 363-372.

Hövelmann, T. 1995: Vegetationskundliche Untersuchungen an den Zwergstrauchheiden Islands. Diss. Bot. 25. - Berlin, Stuttgart.

SснміDт, F.-U. 1991: Ísland. Naturkundlicher Reiseführer 1. - Göttingen.

Schutzbach, W. 1999: Island. Feuerinsel am Polarkreis. - Bonn.

Steinecke K. 1993: Stadtökologie und Umweltschutz in Island. - Norden 9: 31-58.

Thannheiser, D. 1987: Die Pflanzengesellschaften der isländischen Salzwiesen. - Acta Botanica Islandica 9: 35- 60.

Thannheiser, D. 1988: Die Pflanzengesellschaften der isländischen Küstendünen. - Norden 6: 1-12.

Thannheiser, D. 1995: Die Küstenvegetation auf Island und den Färöer-Inseln. - Kölner Geogr. Arbeiten 66: 109-120.

\section{Internetseite}

http://www.welt-in-zahlen.de/index.phtml, Berlin Kania, M. \& Milan, M. 2007: Die Welt in Zahlen. 